\begin{tabular}{|c|c|c|c|c|c|c|c|}
\hline & 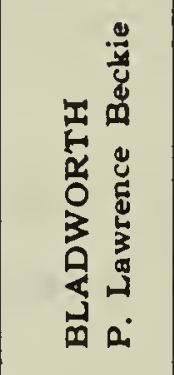 & 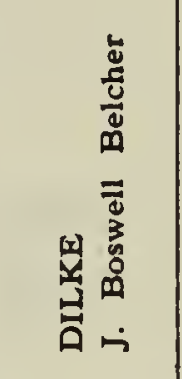 & 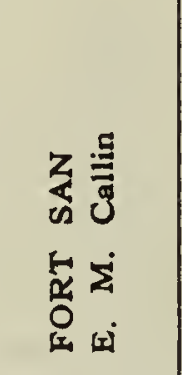 & 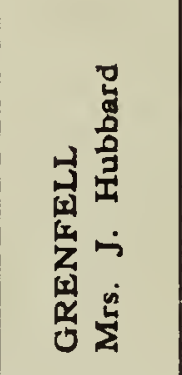 & 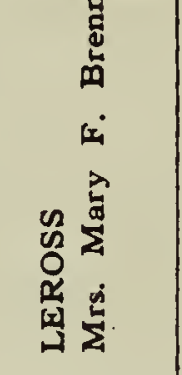 & 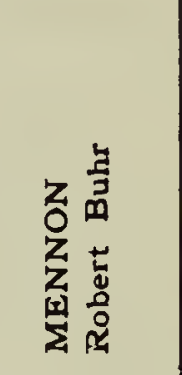 & 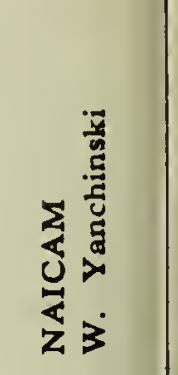 \\
\hline Whistling Swan & Ap. 6 & Ap. 17 & Ap. 6 & Ap. 30 & My. 8 & Ap. 18 & - \\
\hline Canada Goose & Ap. 9 & Ap. 16 & Mr. 21 & Mr. 30 & Ap. 29 & Ap. 19 & Ap. 14 \\
\hline Mallard & Mr. 31 & Ap. 17 & Ap. 7 & Ap. 5 & Ap. 4 & Ap. 6 & Ap. 20 \\
\hline Pintail & Ap. 13 & Ap. 4 & Ap. 5 & Ap. 5 & Ap. 10 & Ap. 17 & Ap. 21 \\
\hline Marsh Hawk & Mr. 31 & Mr. 29 & Mr. 31 & Mr. 27 & Ap. 12 & Ap. 11 & My. 1 \\
\hline Killdeer & Ap. 14 & Ap. 16 & Ap. 5 & Ap. 17 & Ap. 14 & Ap. 21 & Ap. 15 \\
\hline Wilson's Snipe & Ap. 22 & - & My. 5 & My. 14 & 一 & My. 2 & Ap. 26 \\
\hline Mourning Dove & My 11 & My. 10 & Ap. 22 & \begin{tabular}{|l|} 
Jn. 3 \\
\end{tabular} & My. 9 & - & My. 12 \\
\hline Nighthawk & - & - & My. 26 & My. 30 & - & My. 28 & - \\
\hline Ruby-thr Hummingbird & - & - & My. 25 & - & Jn. 15 & - & Jn. 1 \\
\hline Flicker & Ap. 25 & Ap. 20 & Ap. 22 & Ap. 22 & Ap. 22 & Ap. 24 & My. 2 \\
\hline Eastern Kingbird & My. 24 & My 22 & My. 24 & My. 25 & My. 24 & My. 27 & My. 23 \\
\hline Eastern Phoebe & - & - & Ap. 27 & - & - & - & Ap. 26 \\
\hline Barn Swallow & My. 8 & My. 8 & My. 2 & My. 12 & My. 11 & My. 18 & Мy. 9 \\
\hline Purple Martin & My. 27 & - & - & - & - & - & - \\
\hline Crow & Mr. 21 & Mr. 21 & My. 24 & Mr. 24 & Mr. 20 & Mr. 30 & Mr. 18 \\
\hline House Wren & My. 29 & Jn. $\quad 2$ & My. 3 & My. 20 & Jn. 1 & Jn. 14 & My. 8 \\
\hline Catbird & My. 30 & - & My. 25 & My. 26 & Jn. 1 & Jn. 13 & Jn. 2 \\
\hline Brown Thrasher & My. 9 & My. 5 & My. 4 & My. 13 & My. 31 & My. 17 & - \\
\hline Red-eyed Vireo & - & - & My. 26 & - & - & - & My. 6 \\
\hline Black and White Warbler & - & - & My. 19 & - & - & - & 一 \\
\hline Yellow Warbler & My. 13 & My. 14 & My. 12 & My. 23 & My. 24 & Jn. 3 & Jn. 8 \\
\hline Myrtle Warbler & My. 3 & My. 6 & My. 2 & 一 & - & - & My. 15 \\
\hline Ovenbird & - & - & My. 26 & - & - & - & - \\
\hline Redstart & - & - & My. 28 & 一 & - & - & - \\
\hline Red-winged Blackbird & Ap: 5 & Ap. 3 & Ap. 5 & Ap. 5 & My. 27 & Ap. 24 & My. 3 \\
\hline Baltimore Oriole & My. 22 & My. 24 & My. 14 & My. 25 & My. 24 & My. 27 & Jn. 6 \\
\hline Rose-breasted Grosbeak & - & - & My. 14 & - & - & My. 15 & - \\
\hline Goldfinch & My. 23 & My. 27 & My. 11 & My. 26 & Jn. 1 & My. 27 & My. 29 \\
\hline Slate-colored Junco & Ap. 8 & Ap. 21 & Mr. 30 & Mr. 22 & Mr. 30 & Ap. 5 & Ap. 9 \\
\hline Chipping Sparrow. & My. 24 & My. 29 & My. 4 & My. 9 & My. 18 & My. 13 & - \\
\hline White-Crowned Sparrow & My. 9 & My. 5 & My. 7 & My. 4 & My. 10 & My. 12 & 二 \\
\hline White-throated Sparrow & My. 12 & My. 14 & My. 10 & Ap. 27 & My. 4 & My. 3 & My. 12 \\
\hline
\end{tabular}




\section{RATION STUDY，1957}

Compiled by Mary Houston, Yorkton.

\begin{tabular}{|c|c|c|c|c|c|c|c|c|c|}
\hline 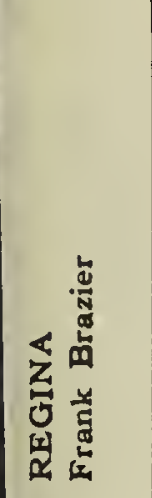 & 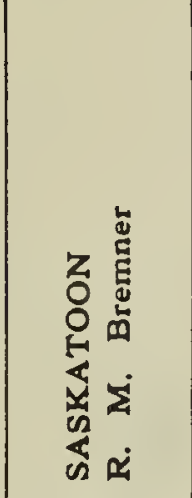 & 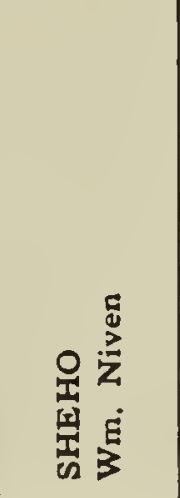 & 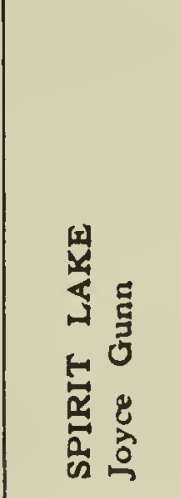 & 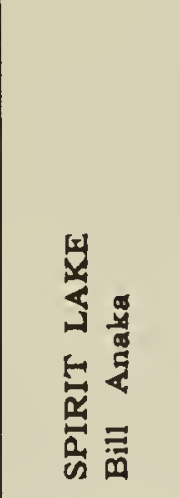 & 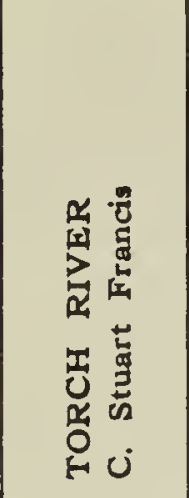 & 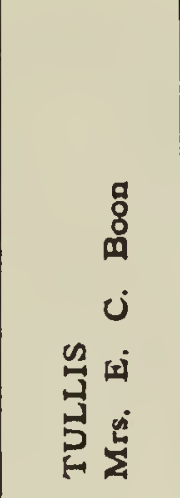 & 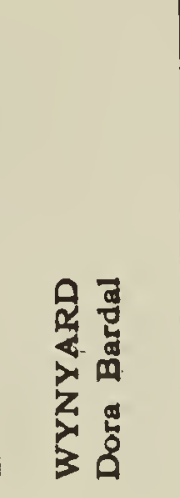 & 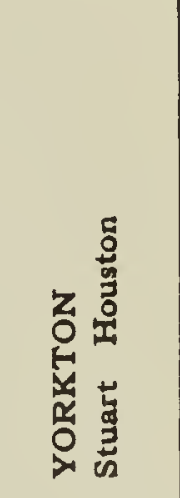 & 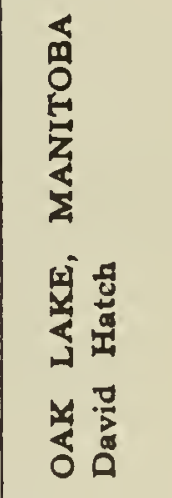 \\
\hline Ap. 20 & Ap. 22 & p. 24 & Ap. 21 & Ap. 21 & - & Ap. 16 & - & Ap. 21 & Ap. 5 \\
\hline Ap. 21 & My. 5 & Mr. 25 & Ap. 14 & Ap. 17 & Ap. 21 & Ap. 16 & Ap. 5 & Mr. 21 & Mr. 21 \\
\hline Ap. 6 & Ap. 14 & Ap. 14 & Ap. 14 & Ap. 17 & - & Ap. 2 & Ap. 7 & Ap. 17 & Mr. 27 \\
\hline Ap. 6 & Ap. 14 & Ap. 14 & Ap. 26 & Ap. 17 & Ap. 24 & Ap. 6 & Ap. 9 & Ap. 17 & Mr. 27 \\
\hline Ap. 5 & Ap. 27 & Ap. 6 & Mr. 28 & Ap. 7 & Ap. 18 & Ap. 16 & Ap. 4 & Ap. 14 & Ap. 1 \\
\hline Ap. 19 & Ap. 22 & Ap. 17 & Ap. 15 & Ap. 15 & Ap. 17 & Ap. 11 & Ap. 16 & Ap. 20 & Mr. 30 \\
\hline - & - & - & Ap. 24 & Ap. 21 & Ap. 12 & - & - & Ap. 28 & Ap. 19 \\
\hline Ap. 30 & My. 12 & My. 4 & Ap. 25 & Ap. 30 & Ap. 30 & Ap. 18 & Мy. 12 & Ap. 28 & Ap. 23 \\
\hline 1y. 30 & Jn. 4 & My. 24 & My. 26 & My. 25 & Мy. 19 & - & - & My. 24 & My. 18 \\
\hline - & $=$ & Jn. 1 & My. 26 & Jn. 17 & Jy. 2 & - & My. 23 & - & Jn. 1 \\
\hline p. -19 & Ap. 28 & Ap. 22 & Ap. 23 & Ap. 21 & Ap. 24 & - & Ap. 23 & Ap. 24 & Ap. 22 \\
\hline 1y. 11 & My. 26 & My. 26 & My. 29 & My. 21 & My. 26 & My. 21 & My. 27 & My. 12 & My. 13 \\
\hline p. 26 & - & Ap. 24 & Ap. 22 & Ap. 21 & Ap. 23 & - & My. 6 & Ap. 28 & - \\
\hline y. 4 & My. 18 & My. 1 & My. 2 & My. 9 & My. 19 & My. 17 & Ap. 29 & My. 6 & My. 3 \\
\hline- & - & My. 26 & My. 21 & My. 18 & Jn. 10 & - & - & Ap. 28 & - \\
\hline Ir. 27 & Ap. 14 & Mr. 24 & Mr. 30 & Mr. 25 & Ap. 3 & Mr. 28 & Mr. 24 & Mr. 17 & Mr. 21 \\
\hline h. 1 & Мy. 18 & My. 16 & My. 7 & My. 7 & Jn. 3 & - & My. 7 & My. 16 & My. 14 \\
\hline 1. 1 & - & My. 24 & My. 27 & My. 27 & - & My. 23 & Мy. 29 & - & My. 24 \\
\hline y. 14 & My. 16 & My. 7 & My. 22 & My. 19 & - & My. 23 & \begin{tabular}{|l} 
My. 29 \\
\end{tabular} & My. 15 & - \\
\hline- & - & My. 21 & - & Jn. 2 & My. 30 & - & - & - & - \\
\hline y. 3 & - & - & - & - & - & - & - & - & My. 25 \\
\hline y. 11 & My. 16 & My. 16 & My. 25 & My. 19 & - & - & My. 26 & My. 26 & My. 13 \\
\hline p. 26 & My. 11 & Ap. 24 & Ap. 28 & Ap. 24 & My. 5 & - & - & My. 7 & My. 6 \\
\hline - & - & - & - & My. 18 & Jn. 28 & - & - & - & - \\
\hline - & - & - & - & - & My. 17 & - & - & - & Jn. 1 \\
\hline b. 14 & Ap. 22 & Ap. 15 & Ap. 14 & Ap. 14 & - & Ap. 25 & - & Ap. 20 & Mr. 26 \\
\hline y. 25 & Jn. 3 & My. 24 & My. 17 & My. 19 & Jy. 3 & My. 24 & My. 27 & My. 24 & My. 24 \\
\hline y. 16 & My. 26 & - & - & My. 19 & My. 22 & - & - & My. 12 & - \\
\hline y. 22 & My. 26 & My. 24 & Jn. 3 & My. 19 & Jn. 1 & - & Jn. 3 & My. 26 & My. 18 \\
\hline r. 31 & Ap. 21 & Ap. 8 & Mr. 28 & Mr. 27 & Ap. 14 & Mr. 27 & Mr. 30 & Mr. 27 & Ap. 3 \\
\hline 4 & My. 16 & My. 14 & My. 7 & My. 12 & Mr. 17 & - & - & My. 11 & My. 5 \\
\hline y. 6 & My. 12 & My. 13 & - & My. 13 & My. 16 & My. 1 & My. 14 & Ap. 28 & My. 6 \\
\hline y. 3 & My. 5 & Ap. 27 & Ap. 30 & Ap. 25 & My. 3 & - & - & My. 5 & My. 1 \\
\hline
\end{tabular}

